\title{
Fractal and Euclidean Geometrical Diagnosis of Cervix Cytology Javier Rodríguez ${ }^{1 *}$, Signed Prieto ${ }^{2}$, Catalina Correa ${ }^{3}$, Fernando Polo ${ }^{4}$ and Paula López ${ }^{5}$
}

${ }^{1}$ Insight Group Director, Investigador de la Fundación Universitaria Ciencias de la Salud. Research Center - Clínica del Country, Bogotá, Colombia ${ }^{2}$ Insight Group researcher. Fundación Universitaria Ciencias de la Salud. Research Center - Clínica del Country, Bogotá, Colombia

${ }^{3}$ Psychologist, Insight Group researcher. Fundación Universitaria de Ciencias de la Salud. Research Center - Clínica del Country, Bogotá, Colombia ${ }^{4}$ Pathologist, Pathology Group Director, Fundación Universitaria de Ciencias de la Salud, Bogotá, Colombia

${ }^{5}$ Department of Medicine, Fundación Universitaria de Ciencias de la Salud, Bogotá, Colombia

\section{Abstract}

Background: Conventional methods for evaluation of cervix cytology show reproducibility problems. To solve this, there was developed a diagnostic methodology based on fractal and euclidean geometry, mathematically differentiating normality, L SIL and H SIL.

Objective: The aim of the present work is to confirm the clinical applicability of such diagnostic in a blind study.

Methods: The clinic diagnosis of 15 normal cells, 15 ASCUS, 15 L SIL and $15 \mathrm{H} \mathrm{SIL} \mathrm{was} \mathrm{masked.} \mathrm{Cellular}$ nucleus and cytoplasm were evaluated calculating fractal dimension, number of spaces occupied by the frontier and number of pixels occupied by the surface of each object. The mathematical diagnosis was established and compared with the conventional diagnosis, calculating specificity, sensibility, negative likelihood ratio and Kappa coefficient.

Results: It was found that simultaneous measures of the nuclear surface and the subtraction between the frontiers of cytoplasm and nucleus, lead to differentiate normality, L SIL and H SIL. Both sensibility and specificity values were of 100 percent. Kappa coefficient was 1 and negative likelihood ratio was zero. 4 ASCUS showed mathematical measures of normality, while the remaining 11 showed values of L-SIL cells.

Conclusion: The mathematical diagnostic prove to be useful for clinical evaluation of cervix cytology, differentiating normality, L SIL and H SIL, quantifying how close it is the cell to a higher severity stage, and clearing up the undetermination of the ASCUS cells.

Keywords: Diagnosis; Fractal; Euclidean; Cervix cancer; Cytology; ASCUS; L-SIL; H-SIL

\section{Introduction}

Due to human body is composed by irregular forms and structures; Euclidean geometry is not enough for its characterization, presenting many limitations. On the contrary, fractal geometry has allowed dealing with the study of human body not only at structural, but at physiological level [1-4]. The measure obtained by the study of natural and abstract objects through fractal geometry is called fractal dimension, and represents the irregularity grade of such object [5]. There are different methods for fractal dimension calculation: abstract fractals as Sierpinski triangle, are properly characterized by the Hausdorff dimension; wild fractals, in which parts are superimposed, are measured by Box Counting method [6,7]; statistical fractals allow to characterize hyperbolic distributions by Zipf-Mandelbrot law [8].

In medicine, fractal geometry application has allowed the approaching to different phenomena as arterial structure [9], cardiac morphophysiology [10-12] and immune repertoire against specific antigens [13]. In oncology field, specifically, fractal geometry has demonstrate its utility for neoplastic detection in mammogram $[14,15]$, in invasive bladder cancer characterization [16], for characterizing different grades of neoplastic disease in oral mucous [17], and for the characterization of vasculature in androgen-dependent tumors [18].

Worldwide, cervix cancer causes about 231.000 deaths yearly, which more than $85 \%$ are countries in process of development, being the second type of cancer more frequent in women. Its annual incidence is about 530000 cases, occupying the fourth place on the more frequent cancer in America [19]. Cervix cytology is the screening technique used par excellence nowadays; it allows the detection of preneoplastic injuries and early therapeutic interventions which decrease mortality associated to this disease [20]. Its diagnostic analysis is based on qualitative techniques dependent of observer experience, requiring a confirmation by a colposcopy and a biopsy [21], demanding additional time and resources. Although it has not been adopted worldwide, Bethesda system is one of the most used methods for qualitative cytology classification [20]. Even though current diagnostic methods present specificity between $98 \%$, they have a sensitivity which varies between $51 \%$ [22], and the false negatives percentage can vary between 20 and $40 \%[23,24]$. This is consequence of the difficulties implied in establishing differences in cells with similar characteristics observed from qualitative methods $[25,26]$.

This system includes diagnostic indeterminacies which are called ASCUS (Atypical Squamous Cells of Undetermined Significance). This qualification includes cells which could not be classified in normality category, or any disease category, which implies a potential danger for the patient. This category presents the less reproducibility within

*Corresponding author: Javier Rodríguez Velásquez, Cra. 79B No. 51-16 Sur Int. 5. Apartment. 102, Kennedy, Bogotá D.C., Colombia, Tel: 057 3134057252; E-mail: grupoinsight2025@yahoo.es

Received January 06, 2014; Accepted February 26, 2014; Published February 28, 2014

Citation: Rodríguez J, Prieto S, Correa C, Polo F, López P (2014) Fractal and Euclidean Geometrical Diagnosis of Cervix Cytology. J Cancer Sci Ther 6: 081086. doi:10.4172/1948-5956.1000253

Copyright: (c) 2014 Rodríguez J, et al. This is an open-access article distributed under the terms of the Creative Commons Attribution License, which permits unrestricted use, distribution, and reproduction in any medium, provided the original author and source are credited. 
observers, constituting the most important diagnostic problem to be solved in this field [27-29]. This situation demands the development of new methods objectively applicable, observer independent and reproducible, allowing an unambiguous morphological diagnostic.

In this context, there was developed a methodology to differentiate mathematically normality and L-SIL, achieving to establish the diagnostic of ASCUS, establishing if they have Normality or L-SIL characteristics. This method is based on fractal dimensions and the application of Intrinsic Mathematical Harmony concept [30]. This research allowed later the finding of all the cellular geometric prototypes of normality, malignancy and the evolution of these states [31].

Recently, there were developed new methodologies based on simultaneous fractal and Euclidean measures, which allow improve the methodologies based only on fractal geometry. This is the case of a new diagnostic method of erythrocyte structure, which allow differentiate normality from disease and so determine viability of bags for transfusion [32]. Also there was developed a methodology which allow establish arterial normality or reestenosis in animal experimentation models, as well as the advance of this pathology [33].

On the context of cervix cytology, there was developed a diagnostic methodology based on fractal and Euclidean geometry, which got differentiate normal, L-SIL and H-SIL cells in an objective and reproducible way. This methodology is based on the quantification of changes in the size of nucleus and surface frontiers, as well as nucleus surface, getting quantify the injury severity increase and clearing the indeterminate character of ASCUS cells, overcoming the reproducibility problems of conventional methodologies, like the Bethesda system.

This research has as goal confirm the clinical capacity of the methodology previously developed, based on fractal and Euclidean geometry, to differentiate cervix cells with different grades of alteration from normality to carcinoma. For this purpose the mathematical diagnosis is compared with the conventional diagnosis by a blind study, establishing sensitivity, specificity, Kappa coefficient and negative likelihood ratio.

\section{Materials and Methods}

\section{Definitions}

Fractal: Term proposed by Benoit Mandelbrot, referred to fragmented or irregular structures or dynamics. Used as a noun, it means "irregularity"; used as an adjective, it means "irregular" [7].

Fractal dimension: Non-integer numerical value that quantifies the irregularity degree of an object or dynamics. As there are different methods to calculate this value, the characteristics of the specific phenomenon studied are the basis to choose the method. The body structures, including cervical cells, are called wild fractals, and are measured by of Box-Counting dimension [7].

Box-counting dimension: It is defined by the eqn (1), establishing a mathematical quantification of the changes in the irregular structure when are observed in different scales [7].

$$
D=\frac{\log N\left(2^{-(K+1)}\right)-\log N\left(2^{-K}\right)}{\log 2^{K+1}-\log 2^{K}}=\log _{2} \frac{N\left(2^{-(K+1)}\right)}{N\left(2^{-K}\right)}
$$

Where:

D: fractal dimension.
$\mathrm{N}$ : Number of squares containing the frontier of the object.

K: Grade of partition of the grid.

Object frontier: Number of squares occupied by the frontiers of the measured object, in the grid of 2 pixel's side (Figure 1).

Object surface: It is defined as the number of pixels occupied by the surface of each one of the measured objects (nucleus and cytoplasm). The software used makes a segmentation process, in order to establish the frontier of the nucleus and the cytoplasm. Next, it counts the number of pixels occupied inside each object, including the pixels of the frontiers (Figure 2).

Substraction of cytoplasm-nucleus frontiers: This value is the result of resting the number of squares occupied by the cytoplasm frontier and the number of squares occupied by the nucleus frontier, in the grid of 2 pixel's side.

\section{Procedure}

There were taken 60 Cervix cytology samples from women between 20-55 years. The samples were obtained from the Pathology Department of Hospital San José. The diagnostic were established by an expert pathologist, following the conventional parameters of Bethesda System, in which H-SIL classification includes carcinoma cells [34]. There were included 15 normal cells, 15 ASCUS, 15 L-SIL and 15 $\mathrm{H}$-SIL. The clinical diagnosis were masked, taking access only to the samples, that were observed with a Leika DM-2500 optical microscope, using a 100X zoom, in order to obtain the Digital pictures. Next, the pictures were analyzed through an image editor.

In order to obtain the mathematical diagnosis, it was used a software previously developed, which superimpose to each image two grids of 2 and 4 pixels, and counts the number of squares occupied by the frontier of each object in each grid, as well as the number of pixels occupied by their surface (see definitions). The fractal dimension of
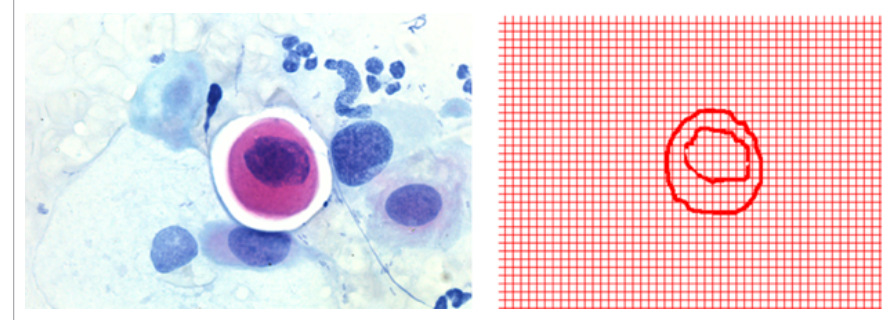

Figure 1: Original image (left), and Image obtained by the software (right), of the nucleus and cytoplasm frontiers of an H-SIL cell. The red lines correspond to the grid of 2 pixel's side.
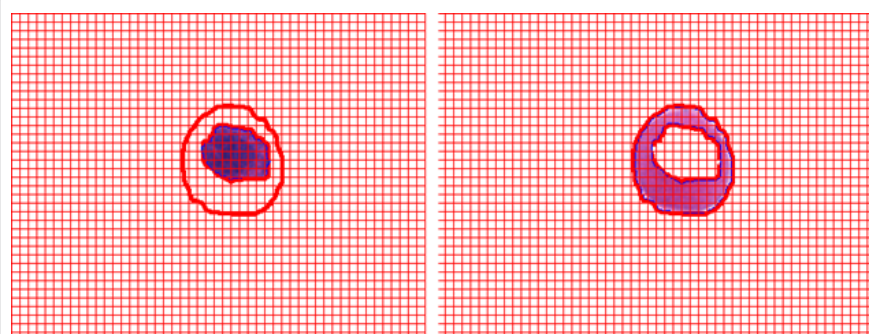

Figure 2: Example of Cytoplasm (left) and nucleus (right) surfaces, of the $\mathrm{H}$-SIL cell observed in figure 1 , evaluated with the software. The red lines correspond to the grid of 2 pixel's side. 
each object is calculated with the number of squares occupied by the object frontier, in the two grids (see eqn (1)).

Based on these measures, it is established the physical-mathematical diagnostic, where normality is characterized by nuclear surfaces less than or equal to 735 pixels and in the rest of the frontiers CytoplasmNucleus must be values greater than or equal to 161 squares in the grid of 2 pixel's side.

L-SIL cells have values greater than or equal to 972 pixels on the nucleus surface, as well as value greater than 130 squares in the grid of 2 pixel's side in the rest of Cytoplasm-Nucleus frontiers.

H-SIL cells present values less than 120 in the rest of CytoplasmNucleus frontiers. It means that in order to differentiate L-SIL and $\mathrm{H}$-SIL states the subtraction of Cytoplasm-Nucleus frontiers must be observed. Besides, there is a range of evolution between L-SIL and H-SIL cells, corresponding to the values between 120 and 130 squares in the grid of 2 pixel's side in the subtraction of Cytoplasm-Nucleus frontiers.

\section{Statistical analysis}

After obtaining the mathematical diagnostic, there was unmasked the clinical diagnostic result of each cytology and these results were taken as the Gold-Standard.

In order to establish a contingency table $2 * 2$ for the comparison between the number of normal and abnormal cases concordant and non-concordant, all the cells classified as L-SIL or H-SIL according to the conventional method, were listed within a single classification as abnormal. Based on this table, sensibility, specificity and negative likelihood ratio were calculated, and the level of concordance between Gold-Standard and physical-mathematical diagnosis was established through Kappa coefficient. The ASCUS cells were excluded of the statistical analysis, as they don't have a specific diagnosis of normality or disease from Gold-standard. However, their mathematical behavior was observed, looking for possible diagnostic relations respect to the normality and disease states, in order to specify quantitative differentiations.

\section{Ethics statement}

This work follows the provisions of Declaration of Helsinki in 1995 , considering the theoretical character of the methodology, and taking in account it is based on non-invasive test previously prescribed, protecting patient's privacy, integrity and anonymity. The present research has a theoretical character and is based on non-invasive test previously prescribed, so informed consent was not necessary. Those reasons made the local ethics committee deemed the work exempt from needing full ethical approval.

\section{Results}

The fractal dimensions of the objects showed values between 0,896 and 1,238; these measures can not differentiate any of the states. The minimum and maximum values for each state are shown in Table 1.

Nucleus surface showed values between 305 and 729 pixels for normality; between 721 and 4677 pixels for ASCUS; between 901 and 5417 pixels for L-SIL and between 540 and 2531 pixels for H-SIL (Table 2).

Measurements of the cytoplasm frontier were between 236 and 524 squares in the grid of 2 pixel's side for normal cells; between 210 and
531 squares in the grid of 2 pixel's side for ASCUS cells; between 231 and 508 squares in the the grid of 2 pixel's side for L-SIL and between 96 and 205 squares in the grid of 2 pixel's side for H-SIL cells (Table 2).

For normality, measures of the nucleus frontier presented values between 38 and 143 squares in the grid of 2 pixel's side; ASCUS between 53 and 137 squares in the grid of 2 pixel's side; the L-SIL between 75 and 147 squares in the grid of 2 pixel's side and the H-SIL between 49 and 113 squares in the grid of 2 pixel's side (Table 2).

Subtracting the measured values of the nucleus and cytoplasm frontiers showed values between 168 and 512 squares in the grid of 2 pixel's side for normality, between 139 and 441 squares in the grid of 2 pixel's side for ASCUS, 133 to 413 squares in the grid of 2 pixel's side for L-SIL and between 40 and 122 squares in the grid of 2 pixel's side for H-SIL (Table 2).

The mathematical diagnostic of the cells showed that all normal, L-SIL and H-SIL cells were correctly diagnosed. 4 ASCUS showed mathematical measures of normality, while the remaining 11 presented values of L-SIL cells. Statistical analysis resulted in sensitivity and specificity of $100 \%$, a likelihood ratio of negative zero, and a Kappa coefficient of 1 .

\section{Discussion}

This is the first research which confirms the diagnostic capacity of the developed methodology based on fractal and Euclidean simultaneous measures for the clinical diagnosis of cervix cytology

\begin{tabular}{|c|c|c|c|c|c|c|c|c|}
\hline Dx. & \multicolumn{2}{|c|}{ NORMAL } & \multicolumn{2}{c|}{ ASCUS } & \multicolumn{2}{c|}{ L-SIL } & \multicolumn{2}{c|}{ H-SIL } \\
\hline & MIN & MAX & MIN & MAX & MIN & MAX & MIN & MAX \\
\hline $\mathbf{N}$ & 0,977 & 1,081 & 1,001 & 1,21 & 0,947 & 1,153 & 1,013 & 1,238 \\
\hline $\mathbf{C}$ & 0,988 & 1,062 & 0,899 & 0,967 & 0,981 & 1,016 & 0,959 & 1,062 \\
\hline $\mathbf{T}$ & 0,896 & 1,012 & 0,913 & 1,138 & 0,939 & 1,195 & 1,082 & 1,177 \\
\hline
\end{tabular}

The fractal dimensions found confirm the previous result, showing it is no possible to establish diagnostic differences with these values evaluated solely

Table 1: Minimum (MIN) and maximum (MAX) values of fractal dimensions for each group of cells. N: Nucleus. C: Citoplasm. T: Total object.

\begin{tabular}{|c|c|c|c|c|c|}
\hline Conv. Dx. & Math. Dx. & NS & NF & CF & CF-NF \\
\hline Normal & Normal & 345 & 43 & 541 & 498 \\
\hline Normal & Normal & 367 & 44 & 402 & 358 \\
\hline ASCUS & Normal & 721 & 53 & 239 & 186 \\
\hline Normal & Normal & 309 & 38 & 434 & 396 \\
\hline Normal & Normal & 625 & 52 & 311 & 259 \\
\hline L SIL & L SIL & 2309 & 106 & 506 & 400 \\
\hline L SIL & L SIL & 3361 & 122 & 346 & 224 \\
\hline L SIL & L SIL & 1512 & 85 & 231 & 146 \\
\hline L SIL & L SIL & 4502 & 144 & 382 & 238 \\
\hline ASCUS & L SIL & 2949 & 137 & 284 & 147 \\
\hline H SIL & H SIL & 2224 & 86 & 197 & 111 \\
\hline H SIL & H SIL & 546 & 51 & 97 & 46 \\
\hline H SIL & H SIL & 1907 & 91 & 180 & 89 \\
\hline H SIL & H SIL & 2045 & 113 & 158 & 45 \\
\hline H SIL & H SIL & 1212 & 72 & 149 & 77 \\
\hline
\end{tabular}

Comparing the conventional diagnosis with the mathematical diagnosis it is observed that the latter correctly diagnose normal, LSIL and HSIL cells, and further provides a quantitative diagnostic for ASCUS cells. There were included 2 ASCUS, in order to show how these cells can show a normal or an L-SIL behavior

Table 2: Measurements of 20 of the studied cells. The surface is expressed in pixels, and the frontier in number of squares in the grill of 2 pixels. Dx. Conventional diagnosis. NF: Nucleus Frontier. NS: Nucleus surface. CF: Citoplasm Frontier. C-N Subtraction of Cytoplasm-Nucleus frontiers. 
(CVV). Calculations obtained showed that the subtraction of nucleus and cytoplasm frontiers values, together with nucleus surface measures, allowed differentiate objectively normality, L-SIL and H-SIL, also quantifying the proximity to a more severe state. The methodology developed overcome the reproducibility problems of current qualitative methodologies, by objective and quantitative measures which take in count geometrical changes in disease evolution, as well as its irregular character. Besides, by this method is possible to establish an objective diagnosis of ASCUS cells, finding that it can present values both normal or L-SIL, solving the indeterminacy problem and constituting a practical measure applicable to clinic, that would allow the unification of current classification systems, with serious clinical implications at preventive level.

The finding of a mathematical self-organization underlying to the changes in cellular structure, allow establish an objective and reproducible diagnostic, applicable to each particular case, independently of statistical or epidemiological analysis, as well as risk factors. I this way, this methodology can facilitate the development of public health policies, as well as a better preventive monitoring at clinic level, detecting more clearly which patients must be carefully assessed in time, given its abnormality grade, established quantitatively $[35,36]$.

Although the measurements obtained confirmed the established limits for each cellular state, due to the developed diagnostic method is based on the quantification of a mathematical order underlying to the changes in cell geometrical structure, studies with higher number of cells could allow refine these limits, without altering the essence of the method.

The underlying hypothesis of this work is there is a geometrical order subjacent to the cellular structure, which allows differentiating the different states from normal to H-SIL cells, by means of the evaluation of the changes of nucleus and cytoplasm. This order must be determined by an appropriate geometry. The fractal geometry measures the characteristic irregularity of cells; however, previous works have demonstrated that fractal dimensions are not enough to differentiate normal and abmormal states in cervix cells, making necessary to establish new concepts to achieve a diagnostic [30,31]. This work confirms that fact, because fractal dimensions did not differentiate normal, L-SIL or H-SIL cells (see Table 1). Besides, fractal geometry does not quantify the changes of the cellular structures sizes during disease progress. On the other side, there has been demonstrated that isolated Euclidean measures applied to irregular objects take to paradoxical results [5]. This work achieved to establish analogous measures to surface and length assessed from Euclidean geometry, in the context of the fractal space of Box Counting. So, it makes possible to take in account simultaneously its irregularity. The Box Counting fractal space evaluates the irregularity in different scales [5-7], so it is possible to establish which scale is significant for establishing diagnostic differences. It was found that the differences could be established with the surface measures evaluated in pixels and the subtraction between the cytoplasm and nucleus frontiers in the 2 pixels grid.

The conventional way of thought and research in medicine is based on different diseases classifications, supported in qualitative measures. In the case on preneoplastic and neoplastic injuries, this took to the current reproducibility problems, with the subsequent difficulty to achieve a consensus [24-27]. These qualitative observations already showed that there were changes on the size of nucleus associated to the appearance of preneoplastic and neoplastic injuries, however, due to there was no quantitative measures of these changes, there was no possible to establish objectively a diagnosis; the qualitative observations complicate the comprehension of the problem and do not allow visualize the total phenomena.

In contrast, the achieved diagnosis was possible thanks to the simplicity of the mathematical language, which allow a geometrical comprehension of the total phenomena of cellular variation where each state from normality to carcinoma, is understood as a particular geometrical state of the total phenomenon, on the same way that the falling of a pencil and the moon movement around Earth are described with the same physical-mathematical law. The establishments of mathematical orders underlying the phenomena diversity allows establish objective diagnosis and predictions independently of classifications and statistical considerations. However these type of studies can be done later to confirm the clinical application of achieved methods from the current medical perspective. In this research the statistical measures made presented the best possible concordance respect to Gold Standard, both in sensitivity, specificity, negative likelihood ratio and Kappa coefficient.

Previous researches had showed that in many cases fractal geometry isolated assessed not allow establish diagnostic differences [10-12], however its analysis with specific mathematical concepts, like Intrinsic Mathematical Harmony and fractal dimension variability for preneoplastic cells case [30,31], allowed establish differences between normality and disease $[9-12,30,31]$. On this context, the developed researches with fractal and Euclidean simultaneous measures for the assessment of erythrocyte structure [32] and the restenosis phenomena [33], showed that it was possible to improve the diagnostic capacity achieved only with fractal measures, giving place to the methodology applied in this work. In the case of erythrocyte structure, there was demonstrate that only by this process was possible to establish diagnostic differences, while on the restenosis phenomena was possible to overcome fractal measures, which only differentiate normality from restenosis, getting a quantification of the restenosis progress level. In this research, there was possible an easier quantification of the phenomenon, which facilitate its practical application at clinical level, getting also a quantification of disease level evolution, which facilitate the monitoring in time.

This research is supported on the form of non-causal thinking underlying the theories of modern physics, as statistical mechanics [37], chaos [38], and Quantum theories [39]. From these perspectives, scientific are not seeking cause-effect relations, due to, as Prigogine explains [38], there is no access to a continuous phenomenon. We only have temporal windows, that is, discrete moments in time, without a necessary causal relation between them. In this sense, the objective is to establish causeless mathematical orders underlying the available information. That is why this method does not take into account factors as age or risk factors, but it focuses on the quantification of geometrical characteristics of the phenomenon.

In this sense, although can be thought that the nucleus variation that support the developed diagnosis, are in concordance with the genetic cancer hypothesis, which point out that a tumor is a consequence of a clone expansion of a genetically mutated cell, in fact, independently of the origin of this variations, the methodology reveals a mathematical order which allow establish diagnostic differences. In this sense, is possible to forecast that is possible to find analogical orders underlying to preneoplastic variation process of other type of cells.

This type of reasoning has allowed the development of diagnosis 
and predictions not only in the processing of diagnostic images of cells and tissues, but also in organs as the heart [10-12]. It has been useful in areas as cardiology [40-42], immunology [43], molecular biology [44], infectology [45] and epidemic predictions too [46]. The obtained results show that medicine can be supported in all of its areas from physical principles, overcoming current medicine problems with physicalmathematical predictions applicable at experimental or clinical level, as well as in the public health field.

\section{Acknowledgements}

Thanks to the Research Division of the Fundación Universitaria de Ciencias de la Salud - Hospital de San José, for funding the Project 71-3743-5, approved in the second convening of the 2012. This paper is one of the results of such Project.

Special thanks to Dr. Guillermo Sánchez, Director of Research Division; to Dr. Magda Alba, Coordinator of Post-graduate Research; to Dr. Yira Diaz, Knowledge Manager; and to Nataly Preciado, Coordinator of Internal Convening, for their support.

\section{Financial Support}

This paper is one of the results of Project 71-3743-5, approved in the second convening of the 2012, by the Research Division of the Fundación Universitaria de Ciencias de la Salud - Hospital de San José.

\section{References}

1. Goldberger AL, Rigney DR, West BJ (1990) Chaos and fractals in human physiology. Sci Am 262: 42-49.

2. Goldberger AL (1996) Non-linear dynamics for clinicians: chaos theory, fractals, and complexity at the bedside. Lancet 347: 1312-1314.

3. Goldberger AL, Amaral LA, Hausdorff JM, Ivanov PCh, Peng CK, et al. (2002) Fractal dynamics in physiology: alterations with disease and aging. Proc Nat Acad Sci U S A 99: 2466-2472.

4. West JW (1990) Fractal physiology and chaos in medicine. World Scientific Singapore.

5. Mandelbrot B (2000) Cuánto mide la costa de Gran Bretaña? In: Mandelbrot B editor. Los Objetos Fractales. Tusquets Eds. S.A., Barcelona: 27-50.

6. Peitgen J (1992) Limits and self-similarity. In: Chaos and Fractals: New Frontiers of Science. Springer-Verlag, New York, USA 135-182.

7. Peitgen J (1992) Length area and dimension. Measuring complexity and scalling properties. In: Chaos and Fractals: New Frontiers of Science. SpringerVerlag, New York, USA 183-228.

8. Mandelbrot B (1972) The Fractal Geometry of Nature. Freeman, San Francisco, USA $341-348$

9. Rodríguez JO, Prieto SE, Correa C, Bernal PA, Puerta GE, et al. (2010) Theoretical generalization of normal and sick coronary arteries with fracta dimensions and the arterial intrinsic mathematical harmony. BMC Med Phys 10: 1 .

10. RodrÃguez J, Prieto S, Correa C, Bernal PÃ, Ivarez L, et al. (2012) DiagnÃ $\tilde{A}^{3}$ stico fractal del ventriculograma cardiaco izquierdo. GeometrÃa fractal de

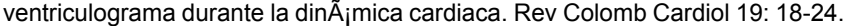

11. RodrÃguez J, Prieto S, Ortiz L, Ronderos M, Correa C (2010) Diagn $\tilde{A}^{3}$ stico Matem $\tilde{A}_{j}$ tico de EcocardiografÃ $\tilde{A s}$ Pedi $\tilde{A}_{j}$ tricas con Medidas de Dimensi $\tilde{A}^{3} n$ Fractal evaluadas con ArmonÃa MatemÃ $i$ tica IntrÃnseca. Rev colomb Cardiol 17: 79-86.

12. RodrÃguez J, Prieto S, Correa C, Bernal P, Tapia D, et al. (2012) Diagn $\tilde{A}^{3}$ stico fractal de disfunci $\tilde{A}^{3} n$ cardiaca severa. Din $\tilde{A}_{j}$ mica fractal de la ramificaci $\tilde{A}^{3} n$ coronaria izquierda. Rev Colomb Cardiol 19: 225-232.

13. RodrÃguez J (2005) Comportamiento fractal del repertorio T especÃfico contra el alergeno Poa P9 (2005) Rev Fac Med Univ Nac Colomb 53: 72-78.

14. Pohlman S, Powell KA, Obuchowski NA, Chilcote WA, Grundfest-Broniatowsk $S$ (1996) Quantitative classification of breast tumors in digitized mammograms. Med Phys 23: 1337-1345.

15. Lefebvre F, Benali H, Gilles R, Kahn E, Di Paola R (1995) A fractal approach to the segmentation of microcalcifications in digital mammograms. Med Phys 22: $381-390$
16. Luzi P, Bianciardi G, Miracco C, De Santi MM, Del Vecchio MT, et al. (1999) Fractal analysis in human pathology. Ann N Y Acad Sci 879: 255-257.

17. Landini G, Rippin JW (1993) Fractal dimensions of the epithelial-connective tissue interfaces in premalignant and malignant epithelial lesions of the floor of the mouth. Anal Quant Cytol Histol 15: 144-149.

18. Gazit Y, Baish JW, Safabakhsh N, Leunig M, Baxter LT, et al. (1997) Fractal characteristics of tumor vascular architecture during tumor growth and regression. Microcirculation 4: 395-402.

19. WHO/ICO Information Centre on HPV and Cervical Cancer (HPV Information Centre). Human Papillomavirus and Related Cancers in Americas. Summary Report 2010.

20. Robbins S (1996) Aparato genital femenino. In: PatologÃa estructural y funcional. McGraw-Hill Interamericana de EspaÃ $\pm a$ S.A., Madrid: 1156-1160.

21. González-Merlo J, González Bosquet E, Biete A, Casanova L, Mestres J (2000) Cáncer de cérvix. In: Ginecología Oncológica. (2ndedn), Masson, Barcelona, Spain

22. Nanda K, McCrory DC, Myers ER, Bastian LA, Hasselblad V, et al. (2000) Accuracy of the Papanicolaou test in screening for and follow-up of cervical cytologic abnormalities: a systematic review. Ann Intern Med 132: 810-819.

23. Subramaniam A, Fauci JM, Schneider KE, Whitworth JM, Erickson BK, et al. (2011) Invasive cervical cancer and screening: what are the rates of unscreened and underscreened women in the modern era? J Low Genit Tract Dis 15: 110-113.

24. National Institutes of Health (1996) NIH Consensus Statement.

25. Schmidt JL, Henriksen JC, McKeon DM, Savik K, Gulbahce HE, et al. (2008) Visual estimates of nucleus-to-nucleus ratios: can we trust our eyes to use the Bethesda ASCUS and LSIL size criteria? Cancer 114: 287-293.

26. Geisinger KR, Vrbin C, Grzybicki DM, Wagner P, Garvin AJ, et al. (2007) Interobserver variability in human papillomavirus test results in cervico vaginal cytologic specimens interpreted as atypical squamous cells. Am J Clin Patho 128: 1010-1014.

27. Smith AE, Sherman ME, Scott DR, Tabbara SO, Dworkin L, et al. (2000) Review of the Bethesda System atlas does not improve reproducibility or accuracy in the classification of atypical squamous cells of undetermined significance smears. Cancer 90: 201-206

28. Stoler MH, Schiffman M (2001) Interobserver reproducibility of cervical cytologic and histologic interpretations: realistic estimates for the ASCUS-LSIL Triage Study. JAMA 285: 1500-1505.

29. Lachman MF, Cavallo-Calvanese C (1998) Qualification of atypical squamous cells of undetermined significance in an independent laboratory: is it useful or significant? Am J Obstet Gynecol 179: 421-429.

30. Rodríguez J (2011) Nuevo método de ayuda diagnóstica con Geometría fractal para células preneoplásicas del epitelio escamoso cervical. Revista UDCA Actualidad \& Divulgación Científica 14: 15-22.

31. Rodríguez J, Prieto S, Correa C, Posso H, Bernal P, et al. (2010) Generalización fractal de células preneoplásicas y cancerígenas del epitelio escamoso cervical de aplicación clínica. Rev Fac Med 18: 173-181.

32. Correa C, RodrÃguez J, Prieto S, Ãlvarez L, Ospino B, et al. (2012) Geometric diagnosis of erythrocyte morphophysiology: Geometric diagnosis of erythrocyte. J Med Med Sci 3: 715-720.

33. RodrÃguez J, Prieto S, Correa C, Polo F, Soracipa S, et al. (2013) Fractal and euclidean geometric generalization of normal and restenosed arteries: fractal and euclidean geometric generalization of arteries. J Med Med Sci 4: 174-180.

34. Lacruz C (2003) Nomenclatura de las lesiones cervicales (de Papanicolau a Bethesda 2001). Rev Esp Patol 36: 5-10.

35. Goldie SJ, Kim JJ, Myers E (2006) Chapter 19: Cost-effectiveness of cervical cancer screening. Vaccine 24: S3/164-170.

36. Vanni T, Legood R, Franco EL, Villa LL, Luz PM, et al. (2011) Economic evaluation of strategies for managing women with equivocal cytological results in Brazil. Int J Cancer 129: 671-679.

37. Tolman R (1979) Principles of statistical mechanics. (1stedn), Dover Publications, New York, USA

38. Fernández A (1990) Introducción. In: Orden y Caos. Scientific American Prensa Científica S.A.: 4-8 
Citation: Rodríguez J, Prieto S, Correa C, Polo F, López P (2014) Fractal and Euclidean Geometrical Diagnosis of Cervix Cytology. J Cancer Sci Ther 6: 081-086. doi:10.4172/1948-5956.1000253

39. Feynman R, Leighton RB, Sands M (1987) Comportamiento cuÃ intico. In: FÃsica Vol 1. Addison-Wesley Iberoamericana S.A., Wilmington 37.1-37.16.

40. Rodriguez J (2012) Proportional Entropy applied to the Clinic Prediction of Cardiac Dynamics. Proceedings of the Innovations in Cardiovascular Interventions (ICl) Meeting

41. Javier Rodríguez, Catalina Correa, Martha Melo, Darío Domínguez, Signed Prieto, et al. (2013) Chaotic cardiac law: Developing predictions of clinical application. J Med Med Sci 4: 79-84

42. Javier Rodríguez, Signed Prieto, Darío Domínguez, Martha Melo, Fernán Mendoza, et al. (2013) Mathematical-physical prediction of cardiac dynamics using the proportional entropy of dynamic systems. J Med Med Sci 4: 370-381.
43. Rodríguez J, Bernal P, Correa C, Prieto S, Benítez L, et al. (2009) Predicción de unión de péptidos de MSA-2 y AMA-1 de Plasmodium falciparum al HLA clase II. Inmunología 28: 115-124.

44. RodrÃguez J, Bernal P, Prieto S, Correa C (2010) Predictions of CD4 lymphocytes' count in HIV patients from complete blood count. BMC Medical Physics 13: 3

45. Rodríguez JO, Prieto SE, Correa C, Pérez CE, Mora JT, et al. (2013) Predictions of CD4 lymphocytes' count in HIV patients from complete blood count. BMC Med Phys 13: 3.

46. Velásquez JO (2010) [A method for forecasting the seasonal dynamic of malaria in the municipalities of Colombia]. Rev Panam Salud Publica 27: 211-218. 